\title{
NOUVELLE
}

\section{Activation \\ par mécano-transduction \\ de la gastrulation \\ en réponse à des fluctuations de formes cellulaires}

Emmanuel Farge
Mécanique et génétique du développement embryonnaire et tumoral, CNRS UMR168 - physico-chimie Curie, Institut Curie, 11, rue Pierre et Marie Curie, 75005 Paris, France.

efarge@curie.fr

> À l'origine de notre propre développement embryonnaire, nous partageons avec l'ensemble des organismes animaux cette étape cruciale du développement depuis plus de 800 millions d'années: la gastrulation. La gastrulation consiste en la formation de larges domaines de tissus qui internalisent l'embryon précoce souvent sous forme de tubes, et qui vont constituer les organes internes de l'animal adulte, tels que le tube digestif, ou le cœur, les muscles, ou les reins et les poumons, pour les organismes les plus complexes [1].

Dans l'embryon de drosophile, le premier de ces tubes, le mésoderme, donnera naissance à tous les organes internes de l'organisme adulte, excepté le tube digestif. II se forme grâce à la stabilisation apicale (à la surface cellulaire extérieure de l'embryon) du moteur moléculaire myosine-II (Myo-II), ayant pour fonction de contracter la surface externe du tissu et ainsi d'induire la courbure du tissu vers l'intérieur pour former le tube mésodermal interne [2]. Le même processus est à l'origine de la formation de l'endoderme postérieur, c'est-à-dire du tube digestif postérieur, qui advient quelques minutes après la formation du mésoderme [3].

Dans le mésoderme, cette contraction se réalise en deux temps. Dans une première étape, les cellules se contractent de façon erratique et instable [4] $(\rightarrow) \mathrm{du}$ fait de l'apparition de «spots de Myo-

$(\rightarrow)$ Voir la Nouvelle de B. Bénazéraf, $m / s$ $n^{\circ} 1$, janvier 2011, page 12
II » instables sur les apex cellulaires. Les apex des cellules du mésoderme subissent en effet des pulsations stochastiques de l'ordre de $20 \%$ de leur taille, c'est-à-dire de 1 micron, leur existence dépendant de l'expression de la protéine Snail. Après un peu plus de 2 minutes de pulsations, les apex cellulaires se contractent de façon stable et coordonnée, résultat de la stabilisation des « spots de Myo-II » qui se mettent en place progressivement sur les apex celIulaires [5]. Cette stabilisation dépend de l'expression de la protéine secrétée Fog, connue pour activer une voie RhoGTPase à l'origine de la stabilisation médio-apicale du moteur Myo-Il et pour être nécessaire à la constriction apicale collective des cellules du mésoderme menant à son invagination [2].

Nous avons montré que les contraintes mécaniques, développées par les fluctuations de formes cellulaires, activent la stabilisation apicale de la protéine Myo-Il et déclenchent le processus actif d'invagination du mésoderme [6]. Pour cela, nous avons étudié un embryon de drosophile présentant une mutation affectant le facteur Snail, dont les cellules du mésoderme ne fluctuent plus, et qui ne présente plus d'activation de l'invagination du mésoderme [7]. Nous avons, dans un premier temps, observé qu'une simple indentation douce ${ }^{1}$ (de 3 à 5 microns) du mésoderme de ce mutant, à l'aide d'une pointe micrométrique

${ }^{1}$ Échancrure peu invasive. micromanipulée, permettait de réintroduire des contraintes mécaniques dans un contexte mutant déficient en pulsations, permettant la stabilisation de la protéine Myo-Il apicale et induisant l'invagination du mésoderme dépendant de Fog, deux processus inexistants dans le mutant de snail $[6,8]$. Si l'on observe l'invagination active de 50 microns de profondeur, plutôt que l'indentation, celle-ci se propage de façon autonome tout le long du mésoderme. En effet, les cellules invaginées déforment les cellules voisines et activent à leur tour mécaniquement leur contraction apicale, ce qui conduit in fine à leur invagination. On observe alors la propagation active d'ondes de contraction le long du mésoderme par un processus de mécanotransduction.

Dans un second temps, un travail collaboratif a été réalisé avec l'équipe de Nora Dempsey à l'institut Louis Néel de Grenoble, et celle de Christine Ménager du laboratoire Phénix à l'université Pierre et Marie Curie. Dans ce travail, nous avons reproduit quantitativement dans le mésoderme, par voie magnétique, les fluctuations de forme des apex cellulaires dont l'amplitude est de l'ordre de 1 micron seulement. Ceci a été réalisé en injectant dans les cellules du mésoderme des liposomes ultra-magnétiques et en approchant, à quelques microns de distance, un réseau de micro-aimants dont la taille individuelle, de 10 microns, est semblable à celle des cellules individuelles. Le champ magnétique local 

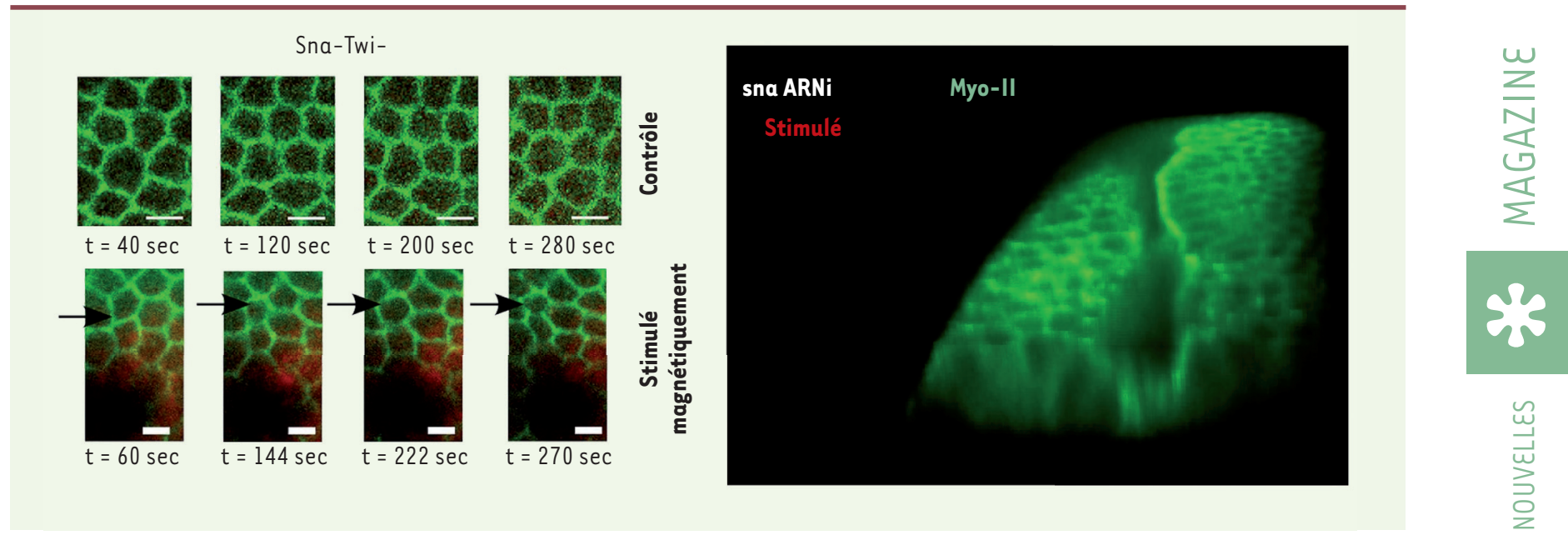

Figure 1. Mimer magnétiquement les pulsations cellulaires dans un embryon déficient rétablit l'invagination du mésoderme. Les pulsations magnétiquement induites (à gauche - en bas) dans le tissu mutant qui ne pulse pas (contrôle; à gauche, en haut) provoquent (à droite) l'invagination active du mésoderme dans l'embryon invalidé par ARN interférence pour l'expression de Snail (mais aussi dans le mutant de snail, non montré) et qui est connu pour ne pas invaginer. Myo-II : myosine II ; Sna : snail ; Twi : twist.

de ces micro-aimants ayant la particularité d'être modulable dans le temps, nous avons fait osciller les champs magnétiques micrométriques locaux, de telle sorte que les apex des cellules pulsent, exactement comme dans un embryon non muté (Figure l-gauche). $\varepsilon n$ réponse à cette stimulation mécanique, nous avons observé la stabilisation apicale du moteur Myo-Il et le déclenchement de l'invagination du mésoderme (Figure 1-droite). Cette stimulation se fait par l'activation mécanique de réactions biochimiques, que nous avons identifiées comme étant la voie de signalisation Fog [6].

Nous avons en effet trouvé, dans le cadre de la stimulation mécanique par indentation, que le mécanisme à l'origine de ce processus est le blocage de l'endocytose de la protéine Fog [8]. Le modèle mécanistique que nous proposons est le suivant: lorsque l'apex d'une cellule se contracte transitoirement, durant la phase stochastique dépendante du facteur de transcription Snail, celle-ci dilate l'apex de la cellule voisine. Elle tend alors sa membrane plasmique et déplie les vésicules d'endocytose, empêchant ainsi la sécrétion de la protéine Fog. Ce blocage a pour effet l'inhibition de la dégradation du complexe Fog-récepteur, normalement internalisé dans les endosomes acides. Conséquence de cette inhibition de l'endocytose, la voie de signalisation est suractivée, aboutissant à la stabilisation apicale du moteur Myo-II. En accord avec ce modèle [9], on observe une accumulation membranaire apicale de la protéine Fog, après deux minutes de fluctuations dépendantes de Snail. Cette accumulation est inexistante chez un mutant du gène codant Snail qui ne fluctue plus. Elle est toutefois observée après stimulation mécanique du mésoderme de ces embryons mutants. Un blocage de l'endocytose immédiat, conditionné à un faible saut de température, dans un embryon doublement muté (pour la dynamine et snail), conduit également au rétablissement de la stabilisation apicale du moteur Myo-II et à l'invagination du mésoderme, comme on l'observe en réponse à la stimulation mécanique [8]. L'inhibition mécanique de l'endocytose comme processus de «mécano-transduction», a également été mise en évidence dans des cultures cellulaires $[10,11]$. In vivo, un mécanisme de mécano-transduction dépendant de la protéine Fog est à l'origine de la stabilisation médio-apicale de la Myo-II qui déclenche l'invagination du mésoderme en réponse aux fluctuations de forme apicales. Toutefois, le rôle de l'inhibition mécanique de l'endocytose de la protéine Fog, démontré en réponse à l'indentation reste à caractériser pour ce qui concerne la réponse aux sollicitations mécaniques pulsées qui miment les fluctuations endogènes physiologiques. Enfin, nous avons également montré, par voie magnétique, que les déformations mécaniques, provoquées par l'invagination du mésoderme sur les cellules de l'endoderme du pôle postérieur de l'embryon (futur tube gastrique postérieur de l'embryon), déclenchent à leur tour la stabilisation apicale de la Myo-Il et initient la formation du tube gastrique selon un processus similaire dépendant de Fog (Figure 2) [6].

Le déclenchement de la gastrulation initie les tout premiers mouvements morphogénétiques actifs de l'embryogenèse chez tous les organismes animaux depuis leur origine. De tout le règne animal, c'est dans l'embryon de drosophile que sa régulation biochimique est la mieux caractérisée. Dans cet embryon, nous avons décrit un processus actif de déclenchement mécaniquement induit 
A

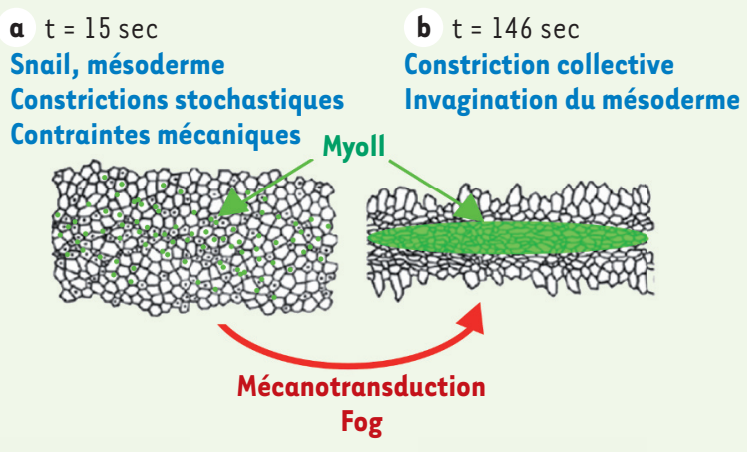

Vue ventrale de l'embryon : mésoderme

B

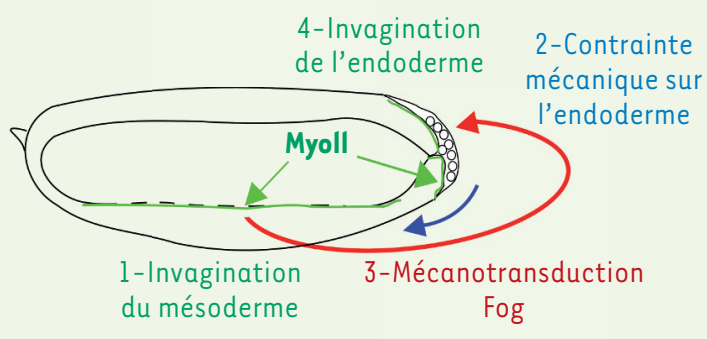

Figure 2. Signalisation mécanique lors de la gastrulation. A. Transition de phase de constriction stochastique/collective induite par mécano-transduction via Fog, à l'origine de l'invagination du mésoderme lors de la gastrulation. B. Cascade auto-inductive des invaginations méso-endodermales de la gastrulation, contrôlée par mécano-transduction dépendant de la protéine Fog.

de la stabilisation apicale de la myosine-II générant l'invagination de l'endoderme et du mésoderme. $\diamond$

Mechanotransduction of gastrulation by cellular fluctuations

\section{LIENS D'INTÉRÊT}

Les auteurs déclarent n'avoir aucun lien d'intérêt concernant les données publiées dans cet article.

\section{RÉFÉRENCES}

1. Gilbert SF, Barresi MJF. Developmental biology. Oxford: Sinauer Associates Inc, 2016 : $810 \mathrm{p}$.

2. Dawes-Hoang RE, Parmar KM, Christiansen AE, et control of myosin localization. Development 2005 ; $132: 4165-78$.

3. Young PE, Pesacreta TC, Kiehart DP. Dynamic changes in the distribution of cytoplasmic myosin during Drosophila embryogenesis. Development 1991 ; $111: 1-14$. al. Folded gastrulation, cell shape change and the
4. Bénazéraf B. Élongation de l'embryon : un mouvement cellulaire aléatoire. Med Sci (Paris) $2011 ; 27: 12-4$.

5. Martin AC, Kaschube M, Wieschaus EF. Pulsed contractions of an actin-myosin network drive apical constriction. Nature 2009 ; $457: 495-9$.

6. Mitrossilis D, Roper JC, Le Roy D, et al. Mechanotransductive cascade of Myo-II-dependent mesoderm and endoderm invaginations in embryo gastrulation. Nat Commun $2017 ; 8: 13883$.

7. Costa M, Sweeton D, Wieschaus $\varepsilon$. Gastrulation in Drosophila: cellular mechanisms of morphogenetic movements. In : Bate M, Martinez-Arias A, eds. The development of Drosophila melanogaster. New York : Cold Spring Harbor Laboratory Press, 1993 : 425-64.

8. Pouille PA, Ahmadi P, Brunet AC, et al. Mechanical signals trigger Myosin II redistribution and mesoderm invagination in Drosophila embryos. Sci Signal 2009 ; 2 : ral6.

9. Driquez B, Bouclet A, Farge $\varepsilon$. Mechanotransduction in mechanically coupled pulsating cells: transition to collective constriction and mesoderm invagination simulation. Phys Biol 2011; 8 : 066007.

10. Diz-Munoz A, Fletcher DA, Weiner OD. Use the force: membrane tension as an organizer of cell shape and motility. Trends Cell Biol $2013 ; 23: 47-53$.

11. Rauch C, Brunet AC, Deleule J, et al. $\mathrm{C} 2 \mathrm{Cl} 2$ myoblast/osteoblast transdifferentiation steps enhanced by epigenetic inhibition of BMP2 endocytosis. Am J Physiol Cell Physiol 2002 ; 283 : C235-43.
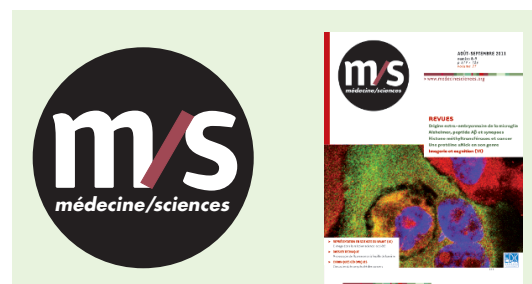

Abonnez-vous

à médecine/sciences

Bulletin d'abonnement page 806 dans ce numéro de $\mathrm{m} / \mathrm{s}$

\begin{tabular}{|c|c|}
\hline $\begin{array}{l}\text { SOCIETE FRANGAISE DE CARCINOLOGIE CERVICO-FACKLE } \\
\end{array}$ & $\begin{array}{l}\text { Bon de commande à retourner à EDP Sciences, 109, avenue Aristide Briand - } 92541 \text { Montrouge Cedex } \\
\text { Tél. : } 0141177405 \text { - Fax : } 0149850345 \text { - E-mail : francois.flori@edpsciences.org }\end{array}$ \\
\hline \multirow{10}{*}{$\begin{array}{c}\text { Pathologie tumorale des glandes salivaires } \\
\text { Préservation salivaire } \\
\text { et nouvelles techniques de radiothérapie }\end{array}$} & 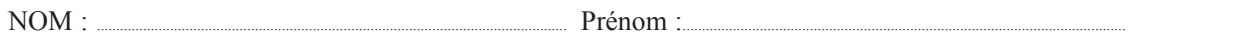 \\
\hline & Adresse : .............. \\
\hline & 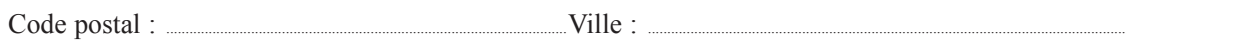 \\
\hline & Pays : \\
\hline & 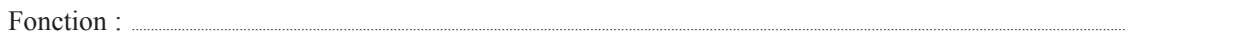 \\
\hline & Je souhaite recevoir l'ouvrage Pathologie tumorale des glandes salivaires : $35 €+3 €$ de port $=\mathbf{3 8} € \mathbf{T T C}$ \\
\hline & Par chèque, à l'ordre de EDP Sciences \\
\hline & $\square$ Par carte bancaire : $\quad \square$ Visa \\
\hline & 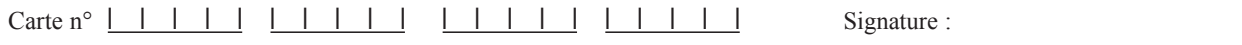 \\
\hline & Date d'expiration: $\quad \underline{|l| l|l|}$ \\
\hline & 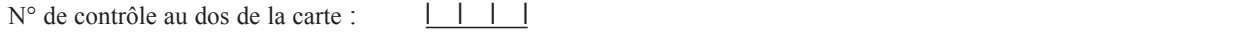 \\
\hline
\end{tabular}

